\title{
Assessment of the Correlation between the Implant Distance and Primary Stability by Resonance Frequency Analysis
}

\author{
Samuel Jeu, ${ }^{1}$ Etienne Guillaud, ${ }^{2}$ Laurent Hauret, ${ }^{1}$ Jean-Christophe Coutant, ${ }^{1,3}$ \\ and Bruno Ella ${ }^{1,3}$ \\ ${ }^{1}$ Department of Odontology and Buccal Health, Bordeaux University, Bordeaux, France \\ ${ }^{2}$ CNRS-UMR 5287, Bordeaux University, Bordeaux, France \\ ${ }^{3}$ Laboratory of Anatomy, Bordeaux University, Bordeaux, France
}

Correspondence should be addressed to Bruno Ella; bruno.ella@u-bordeaux.fr

Received 9 April 2015; Accepted 3 June 2015

Academic Editor: Heng-Li Huang

Copyright (C) 2015 Samuel Jeu et al. This is an open access article distributed under the Creative Commons Attribution License, which permits unrestricted use, distribution, and reproduction in any medium, provided the original work is properly cited.

\begin{abstract}
Objective. The aim of this study was to assess the influence of the interimplant distance on the implant primary stability (ISQ) by Resonance Frequency Analysis (RFA). Method. Forty-five implants were placed in the mandible of human cadavers and 108 in artificial bone substrates in the form of polyurethane foam blocks. Primary implant stability was successively measured first by RFA immediately after the placement of the first implant (A) and then after two other implants (B and C) proximal and distal to the first implant. The interimplant distances were defined from 1 to $6 \mathrm{~mm}$ and the three primary stability values measured were compared. Results. On the mandibles, no correlation was observed between the interimplant distances and primary stability. On the polyurethane foam block, the primary stability of implant A increased significantly $(p<0.001)$ after the placement of implant $\mathrm{B}$ but remained constant after placement of implant C. Conclusion. Reducing the interimplant distance does not affect the primary stability on dry bone or artificial substrate.
\end{abstract}

\section{Introduction}

Primary stability is defined as the degree of mechanical anchoring established when placing an implant [1]. It reflects the resilience of the implant to axial, lateral, and rotational forces and is a fundamental parameter that contributes to the success of the osseointegration of implant [2].

As a whole, implant primary stability is affected by three interrelated factors:

(i) The amount of bone and quality of bone implant contact are important, as described by Gomes de Oliveira et al. [3]; primary stability is greater in a higher density bone than in a low density one. Also, bone cortex thickness highly affects primary stability [4-6].

(ii) Implant morphology (diameter, shape, and macrogeometry) plays an effective role on the primary stability as shown by Coelho et al. [7] and Krafft et al. [8]. (iii) Surgical procedure to prepare the implant bed (thread tapping, subpreparation, or use of an osteotome) is also correlated with the implant primary stability [7, 8]. So, to optimize the implant primary stability, the characteristics of each of these factors must be well used.

The implant primary stability depends on the quality and quantity of peri-implant bone; then reducing the interimplant distance involves a decrease in the amount of interimplant bones. According to Tarnow et al. [9] and Kupershmidt et al. [10], the minimum interimplant distance is set at $3 \mathrm{~mm}$. Below $3 \mathrm{~mm}$, a bone lysis of the interimplant spectrum occurs. The reduction of this distance to $2 \mathrm{~mm}$ has been previously described in the literature by Elian et al. [11], Hermann et al. [12], and Atieh et al. [13], but it consists of reducing the diameter of the abutment compared to the diameter of the implant collar. According to Maeda et al. [14], the gap between the implant collar and the abutment should 
allow movement at the bone implant-abutment junction and protection of the biological space [15].

To date, no study has focused on the biomechanical influence of the interimplant distance on the primary stability. The objective of this study was to assess the correlation between the interimplant distance and the implant primary stability by RFA using the ISQ values. It was of interest to provide a biomechanical approach contributing to define the minimum interimplant distance. The assumption was that the reduction of the interimplant distance would decrease their primary stability.

\section{Material and Methods}

We used natural human bone and an artificial material whose characteristics are similar to human bone.

2.1. Natural Bone. Fifteen fully toothless hemimandibles were taken from embalmed cadavers (fixed in 10\% formalin), with a mean age of 75 years ( \pm 10 years), regardless of gender, at the Laboratory of Anatomy of Bordeaux University. Mandibles with fracture sequelae, any deformities, implants, or bone reconstructions (plaque, screws), were excluded and all soft tissues were removed.

2.2. Artificial Bone. A laminated polyurethane foam block of $120 \times 170 \times 42 \mathrm{~mm}$, meeting the ASTM F-1839-08 norms was used (model 1522-399, Sawbones, Pacific Research Laboratories, US). The block body and surface had two different densities, simulating the mandibular posterior bone and its cortex $[16,17]$ :

(i) The block body was composed of 20 pcf polyurethane foam corresponding to a density of 850 Hounsfield units (HU), simulating the posterior mandibular trabecular bone.

(ii) The block surface was laminated with a $2 \mathrm{~mm}$ thick inflexible $30 \mathrm{pcf}$ polyurethane sheet, corresponding to a density of $1250 \mathrm{HU}$, simulating the posterior mandibular cortical bone.

2.3. Implants. Artificial Mk III Groovy implants (Brånemark System, Nobel Biocare, Sweden) of $3.75 \mathrm{~mm}$ in diameter and $13 \mathrm{~mm}$ in length were used. These implants are recommended by the producer for their anchoring qualities in bone, due to their cylindroconical shape with parallel sides, a standard straight collar, and an external hexagonal connection.

\subsection{Determination of the Implant Sites}

2.4.1. In Natural Bone. Radiopaque markers with Guttapercha cones were fixed on each hemimandibular angle. The CT-scan was performed (Lightspeed PRO 32, GE, Germany). All hemimandibles were maintained in the real acquisition position using a stabilizing base. Using radiopaque markers, 45 implant sites were defined on the CT-scan acquisition with the Simplant Pro software (Materialise, version 14, Leuven, Belgium). Three implant sites were defined on each

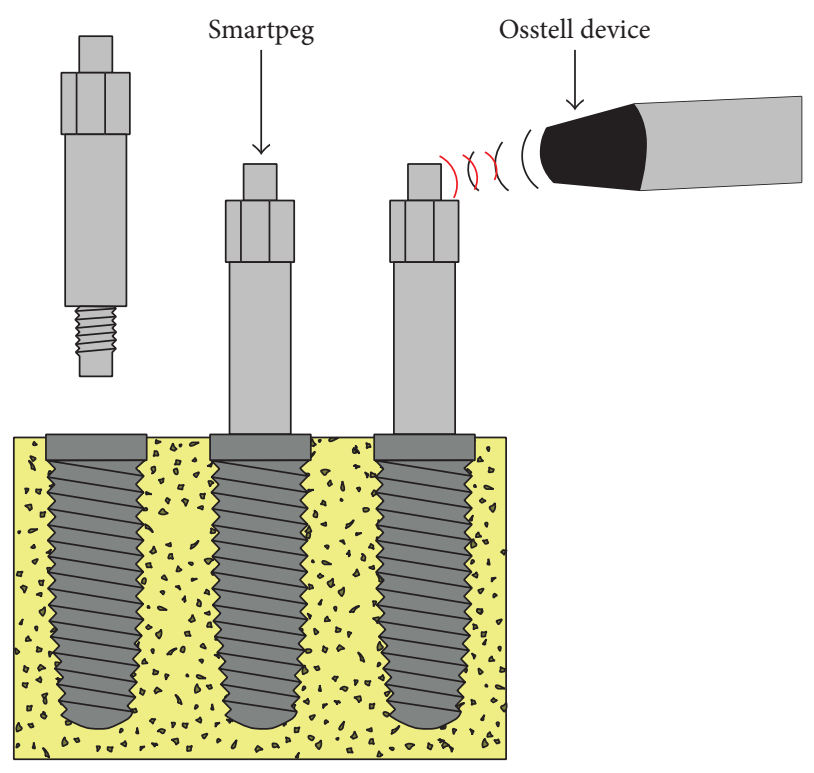

Figure 1: Osstell ISQ measurement procedure.

hemimandible: A, B (proximal sites), and C (distal site). Sites $\mathrm{A}$ and $\mathrm{B}$ were equidistant (distance $Y$ ) from site $\mathrm{C}$ :

(i) on 3 hemimandibles, the intersite distance $Y$ was $1 \mathrm{~mm}$;

(ii) on 4 hemimandibles, the intersite distance $Y$ was $2 \mathrm{~mm}$;

(iii) on 4 hemimandibles, the intersite distance $Y$ was $3 \mathrm{~mm}$;

(iv) on 2 hemimandibles, the intersite distance $Y$ was $4 \mathrm{~mm}$;

(v) on 2 hemimandibles, the intersite distance $Y$ was $5 \mathrm{~mm}$.

The bone density (expressed in HU) and cortical thickness of the implant sites were measured with the Simplant Pro software.

2.4.2. In Artificial Bone. Six territories were determined on the polyurethane foam block. Each territory included six areas where three implant sites were defined: A, B (proximal sites), and C (distal site). Sites A and B were equidistant (distance $Y$ ) from site $C$. An intersite distance $Y$ of 1, 2, 3, 4, 5, or $6 \mathrm{~mm}$ corresponded to each territory and 108 implant sites were defined.

2.5. Implant Placement. Forty-five implants were placed on the mandibles and 108 on the polyurethane block. All implants were placed by the same practitioner. The drilling for each bone type was performed using a single procedure. An Implanteo motor (Anthogyr, France) was used at a rotational speed of $1500 \mathrm{rev} / \mathrm{min}$, with a drilling sequence of successive diameters of $\varnothing 2.0, \varnothing 2.4 / \varnothing 2.8$, and $\varnothing 3.2$ at the length of each implant. The tactile sensation of bone density was recorded by the practitioner during the pilot drilling. 


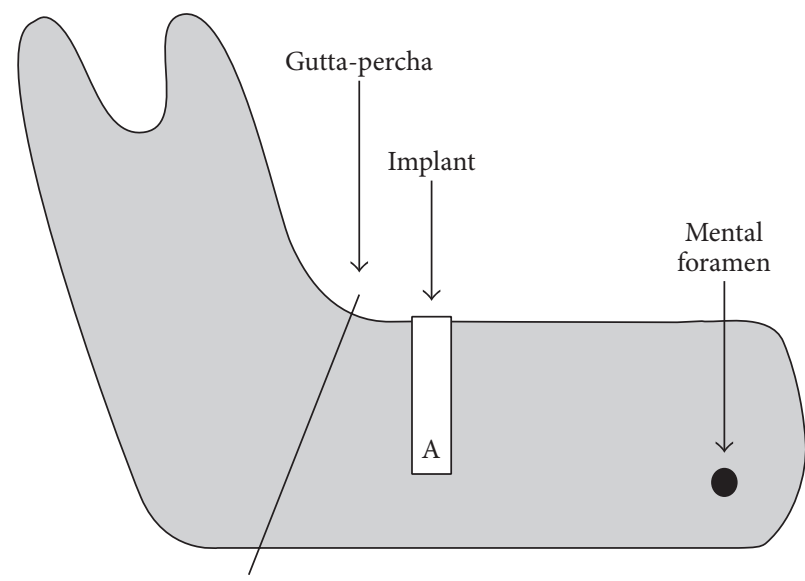

(a)

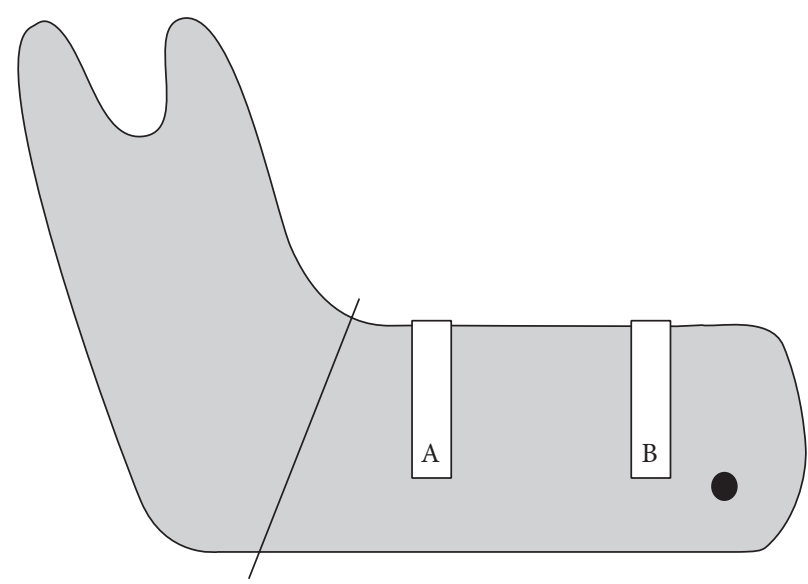

(b)

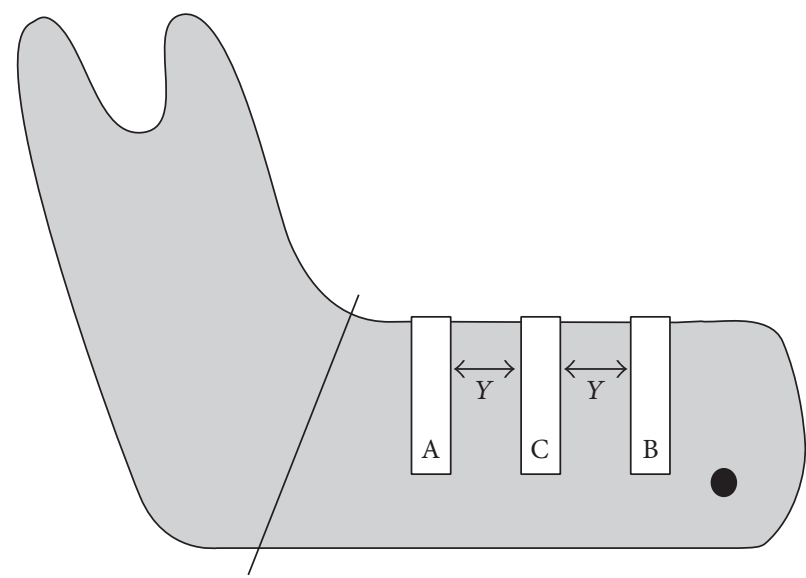

(c)

Figure 2: (a) Placement of implant A. (b) Placement of implant B. (c) Placement of implant C.

Implants $\mathrm{A}, \mathrm{B}$, and $\mathrm{C}$ were placed parallel to each other and perpendicular to the support, at a maximum torque of $40 \mathrm{Ncm}$.

2.6. Measurement of Primary Stability. The Osstell ISQ (Implant Stability Quotient) device accurately and objectively measures the primary stability of an implant placed in both natural and artificial bones [18, 19]. The Osstell device is a digital probe emitting electromagnetic pulses to measure the implant stability by RFA of a SmartPeg screwed on the implant.

Immediately after the implant placement, a SmartPeg Osstell type 1 was manually screwed (Figure 1). The measurements of the associated buccolingual and mesiodistal primary stabilities (ISQBL and ISQMD) were recorded. The mean of these two measurements corresponded to the overall implant primary stability. To verify the reproducibility, all measurements were performed twice.

2.7. Placement and Measurement Chronology. The following placement and measurement chronology was applied in natural and artificial bones.
2.7.1. Implants $A$ and B. Firstly, implant $A$ was placed and the measurements of its ISQ on buccolingual side (ISQA1VL) and mesiodistal side (ISQA1MD) were performed. Secondly, implant $\mathrm{B}$ was placed according to the protocol described above, and its ISQ measurements on buccolingual (ISQB1VL) and mesiodistal (ISQB1MD) sides were also performed (Figures 2(a) and 2(b)).

To ensure that implant B placement did not modify implant A primary stability, implant A ISQ on buccolingual and mesiodistal sides were measured again.

2.7.2. Implant $C$. Implant $C$ was placed equidistantly (distance $Y$ ) from implants A and B (Figure 2(c)). After implant $\mathrm{C}$ placement, a second primary stability of implants A (ISQA2VL, ISQA2MD) and B (ISQB2VL, ISQB2MD) was measured. Then the primary stability of implant C (ISQCVL and ISQCMD) was also measured on both sides.

2.8. Statistical Analysis. The statistical analysis was performed using the statistical software Matlab version R2012b, Natick, USA. An alpha-error probability of $p<0.05$ was adopted as the statistically significant level to determine the correlation between the primary stability and the multiple 


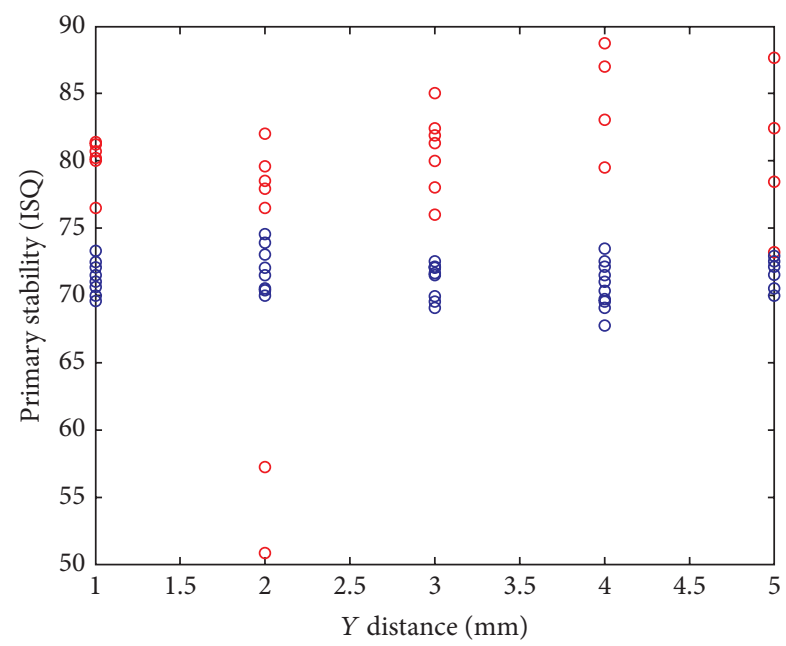

FIGURE 3: Data visualization: postoperative primary stabilities of implants A and B (blue: implant primary stability in polyurethane foam; red: implant primary stability in human mandible).

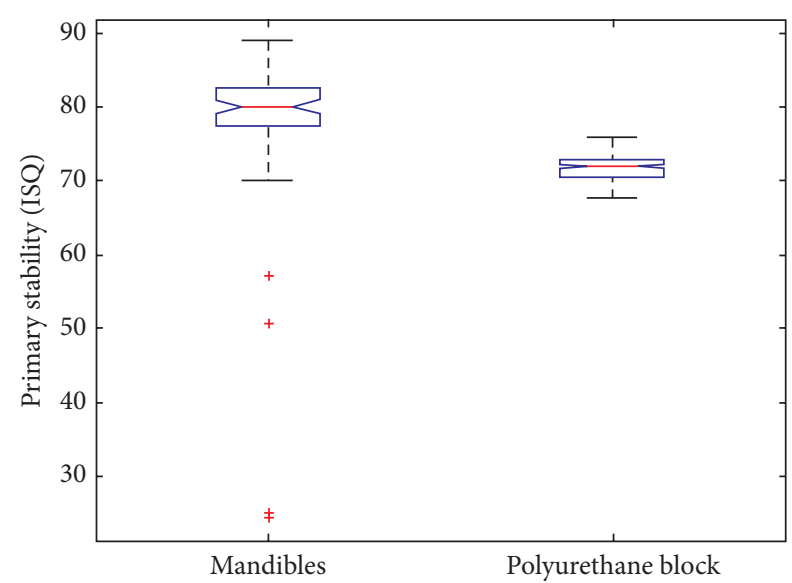

FIGURE 4: Postoperative primary stabilities depending on the support type.

variables identified. Nonparametric tests (Kruskal-Wallis) were used to compare medians of the groups.

\section{Results}

We compared the primary stability on each type of bone substrate (natural and artificial) such as the comparison of implant A stability before and after the placement of implant $\mathrm{B}$ and the comparison of the stability of implants $\mathrm{A}$ and $\mathrm{B}$ before and after the placement of implant C (Figure 3).

3.1. Primary Implant Stability Depending on the Bed Type (Human Mandible and Polyurethane Foam). Differences in variability, normality, and mean (Figure 4) allowed separate testing of the effects on the mandibles and on the polyurethane foam block.
3.2. Comparison of the Primary Implant Stabilities on Human Mandibles. No effect was observed on the primary stability of implant A after the placement of implant B (Figure 5(a)).

After the placement of implant $C$, no effect was observed on the primary stability of implants A and B (Figure 5(b)).

3.3. Comparison of the Primary Implant Stabilities on the Polyurethane Foam Block. In the polyurethane foam, the value of primary stability (ISQ) of implant A was significantly higher $(p<0.001)$ after the placement of implant B (Figure 6(a)).

However no effect was observed on the primary stability of implants A and B after the placement of implant C (Figure 6(b)).

\section{Discussion}

The results of this study demonstrated that reducing the interimplant distance does not affect their primary stability (Figures 5(b) and 6(b)).

Based on a biological approach, Tarnow et al. [9], Kupershmidt et al. [10], and Elian et al. [11] have shown that the minimum interimplant distance currently recommended is of $3 \mathrm{~mm}$. Reducing the minimum interimplant distance implies a decrease in the bone amount between the implants. Therefore, the biomechanical constraints of the interimplant distance on their primary stability was of interest to investigate.

In our study, no correlation was observed between the interimplant distances assessed and the implant primary stability (Table 1). Indeed, bringing the implants closer involved a decrease in the amount of interimplant bone, but it did not affect the amount of bone directly in contact with the implant. Hsu $[20,21]$ showed that the surface contact at the bone implant interface is a major factor for its primary stability. The quantity of bone at the bone implant interface is closely related to the juxtaimplant bone density. On the other hand Ohashi et al. [22] described the biomechanical role played by peri-implant bone. Matsunaga et al. [23] performed analysis using an FE model that closely reproduced trabecular structure in three dimensions and reported that the periimplant trabecular structure is closely connected with its biomechanical role. Bernhardt et al. [24] demonstrated that the analysis of the bone implant volume (BIV) gives information about the thickness of newly formed peri-implant bone, which is of interest in correlation to the determined bone implant contact (BIC). Differences in bone volume are of interest when comparing different implant surfaces and their possible influence on bone formation [25]. The proximity of the implants could cause a peri-implant bone condensation and thus promote the primary stability. Wirth et al. [26] proposed that the influence of the implant primary stability should be attributed to the juxtaimplant bone quality.

Other factors influence the primary stability. Indeed, on the human mandibles, a significant correlation was observed between the bone density and implant primary stability (Table 1). Seong et al. [27] have shown that the bone density and the thickness of the cortical bone can vary rapidly from 


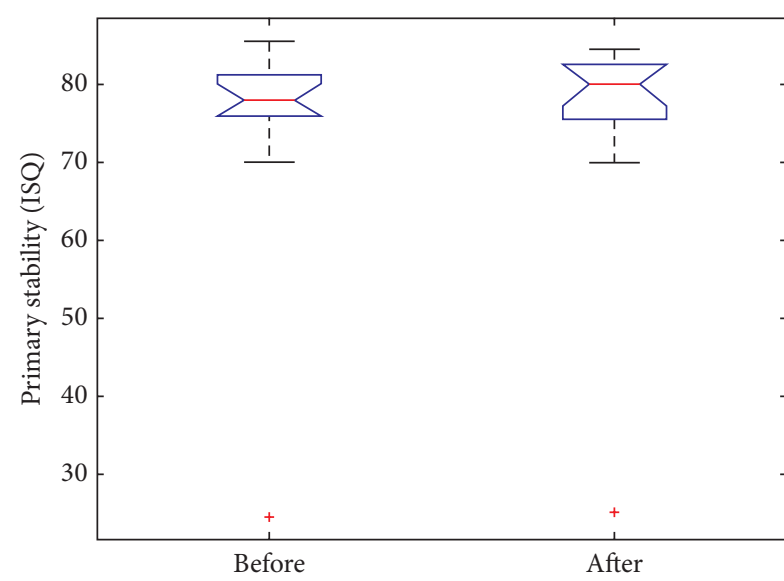

(a)

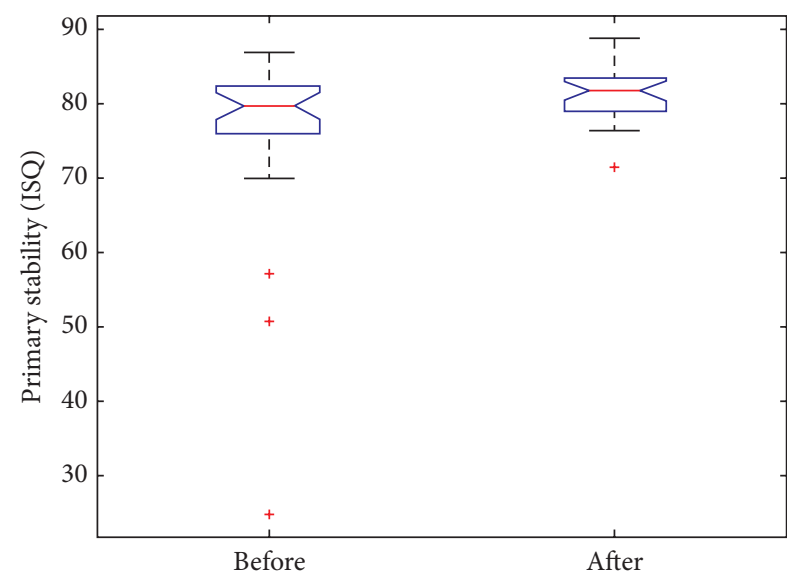

(b)

Figure 5: (a) Comparison of implant A primary stability between before and after the placement of implant B. (b) Comparison of implants $\mathrm{A}$ and $\mathrm{B}$ primary stability between before and after the placement of implant C.

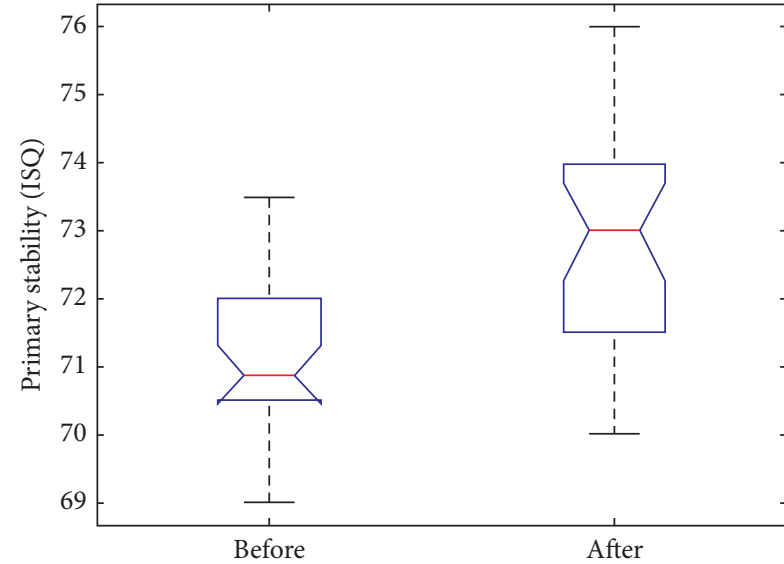

(a)

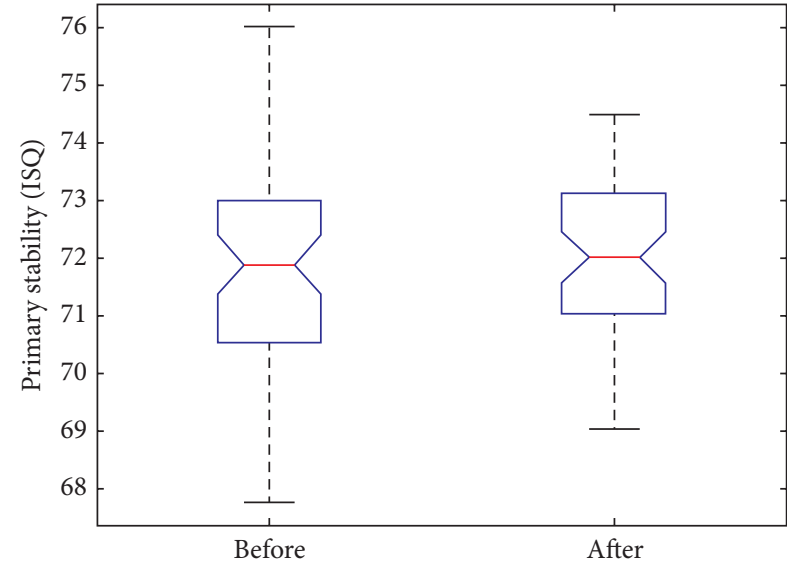

(b)

Figure 6: (a) Comparison of implant A primary stability between before and after the placement of implant B. (b) Comparison of implants A and B primary stability between before and after the placement of implant C.

one human mandible to another and also vary from one site to another on the same human mandible. Due to the fact that this variability can be observed within the same site, it justifies the choice of an area. Otherwise, Hsu et al. [28] have shown a specific sensitivity of the Osstell device to these variability factors of bone density.

Despite the reproducibility of the protocol on the two substrates, a direct comparison was not possible between human dry bone (mandible) and polyurethane foam because of their different properties.

However, the implant stability varied between the polyurethane foam block and the human mandibles (Figure 4). Implant stability was significantly improved on the human mandibles compared with the polyurethane foam block, but implant stability was also much more variable. Implant B placement on the polyurethane foam block increased implant A primary stability (Figure 6(a)) unlike what was observed on the human mandibles (Figure 5(a)).
The polyurethane foam block and the mandibular bone have different mechanical properties that could explain these results. Their elastic properties differ, so, according to van Eijden [29], the transmission of the constraints varies between a heterogeneous anisotropic material such as the mandibular bone and a homogeneous isotropic material such as the polyurethane foam block.

Linde and Sorensen [30] showed bone mechanical properties undergo postmortem change and can also be affected by the storage conditions and duration. The human mandibles taken were stored at $21^{\circ} \mathrm{C}$ on average.

The implant site drilling was performed without irrigation. Overheating the polyurethane could cause a thermal dilation around the implant, responsible for the increase in primary stabilities in this substrate. In addition, the polyurethane foam block density was at $850 \mathrm{HU}$, whereas the human mandibular body average density was at $540 \mathrm{HU}$. Because of this difference, an objective comparison 
TABLE 1: Correlation analysis $($ alpha $=0.05)$.

\begin{tabular}{|c|c|c|c|c|}
\hline Type & Variable 1 & Variable 2 & $R$ & $p$ \\
\hline Hemimandible & Distance $Y$ & Primary stability & 0.122 & 0.252 \\
\hline Hemimandible & Density & Primary stability & 0.271 & 0.010 \\
\hline Hemimandible & Cortical bone & Primary stability & 0.162 & 0.128 \\
\hline Hemimandible & Tactile sensation & Primary stability & -0.185 & 0.081 \\
\hline Hemimandible & Torque & Primary stability & 0.175 & 0.099 \\
\hline Polyurethane foam & Distance $Y$ & Primary stability & -0.112 & 0.136 \\
\hline
\end{tabular}

This table shows the variables that can modify the primary implant stability. Bone density is the only variable correlated to the primary stability $(p=0.01)$ in the natural bone and the artificial bone.

could not be made between the results of these two implant substrates.

There are some limitations associated with the current study.

According to Eliasa et al. [31] and O'Sullivan et al. [32], the implant geometry is one of the factors influencing its primary stability. So the use of only one implant type in our study could have limited the results.

As another limitation, the density variability within the mandibular bone could also be a bias factor.

To limit this disadvantage of heterogeneity of the mandibular bone, we used a polyurethane foam block. This substrate allowed comparison of the primary stability measured without being influenced by the density variability factor from one site to another.

O'Mahony et al. [33] have shown that factors such as blood, heterogeneity, or anisotropy as well as the morphology are likely to influence the measurements; thus the results of the polyurethane cannot be extrapolated to the natural bone. However these results allow understanding the mechanical influence of interimplant distance on implant primary stability.

In conclusion, this study has demonstrated that the reduction of the interimplant distance does not affect the implant primary stability in a dry human bone. However, since the conditions of the living bone were not taken into account in this experiment, it would be interesting to reproduce this study in vivo to assess the influence of the interimplant distance on their primary stability and the evolution of this stability after implant placement, during peri-implant bone healing.

\section{Conflict of Interests}

The authors declare that there is no conflict of interests regarding the publication of this paper.

\section{Acknowledgments}

The authors thank Jean-Jacques, José, André, Bernard, and Etienne for their technical expertise and help in harvesting the fresh cadaver samples for this research in the Laboratory of Anatomy of Bordeaux University, France.

\section{References}

[1] F. Javed and G. E. Romanos, "The role of primary stability for successful immediate loading of dental implants. A literature review," Journal of Dentistry, vol. 38, no. 8, pp. 612-620, 2010.

[2] T. Albrektsson, G. Zarb, P. Worthington, and A. R. Eriksson, "The long-term efficacy of currently used dental implants: a review and proposed criteria of success," The International Journal of Oral \& Maxillofacial Implants, vol. 1, no. 1, pp. 11-25, 1986.

[3] R. C. Gomes de Oliveira, C. R. Leles, C. Lindh, and R. F. Ribeiro-Rotta, "Bone tissue microarchitectural characteristics at dental implant sites. Part 1: identification of clinical-related parameters," Clinical Oral Implants Research, vol. 23, no. 8, pp. 981-986, 2012.

[4] J.-Y. Hong, J.-S. Lee, E.-K. Pang, U.-W. Jung, S.-H. Choi, and C.K. Kim, "Impact of different synthetic bone fillers on healing of extraction sockets: an experimental study in dogs," Clinical Oral Implants Research, vol. 25, no. 2, pp. e30-e37, 2014.

[5] J. Hong, Y.-J. Lim, and S.-O. Park, "Quantitative biomechanical analysis of the influence of the cortical bone and implant length on primary stability," Clinical Oral Implants Research, vol. 23, no. 10, pp. 1193-1197, 2012.

[6] I. Miyamoto, Y. Tsuboi, E. Wada, H. Suwa, and T. Iizuka, "Influence of cortical bone thickness and implant length on implant stability at the time of surgery-clinical, prospective, biomechanical, and imaging study," Bone, vol. 37, no. 6, pp. 776780, 2005.

[7] P. G. Coelho, C. Marin, H. S. Teixeira et al., "Biomechanical evaluation of undersized drilling on implant biomechanical stability at early implantation times," Journal of Oral and Maxillofacial Surgery, vol. 71, no. 2, pp. e69-e75, 2013.

[8] T. Krafft, W. Winter, M. Wichmann, and M. Karl, "Applicability of strain measurements on a contra angle handpiece for the determination of alveolar bone quality during dental implant surgery," Journal of Cranio-Maxillofacial Surgery, vol. 40, no. 5, pp. e144-e149, 2012.

[9] D. P. Tarnow, S. C. Cho, and S. S. Wallace, "The effect of interimplant distance on the height of inter-implant bone crest," Journal of Periodontology, vol. 71, no. 4, pp. 546-549, 2000.

[10] I. Kupershmidt, L. Levin, and D. Schwartz-Arad, "Inter-implant bone height changes in anterior maxillary immediate and nonimmediate adjacent dental implants," Journal of Periodontology, vol. 78, no. 6, pp. 991-996, 2007.

[11] N. Elian, M. Bloom, M. Dard, S.-C. Cho, R. D. Trushkowsky, and D. Tarnow, "Effect of interimplant distance (2 and $3 \mathrm{~mm}$ ) on the height of interimplant bone crest: a histomorphometric evaluation," Journal of Periodontology, vol. 82, no. 12, pp. 17491756, 2011. 
[12] F. Hermann, H. Lerner, and A. Palti, "Factors influencing the preservation of the periimplant marginal bone," Implant Dentistry, vol. 16, no. 2, pp. 165-175, 2007.

[13] M. A. Atieh, H. M. Ibrahim, and A. H. Atieh, "Platform switching for marginal bone preservation around dental implants: a systematic review and meta-analysis," Journal of Periodontology, vol. 81, no. 10, pp. 1350-1366, 2010.

[14] Y. Maeda, J. Miura, I. Taki, and M. Sogo, "Biomechanical analysis on platform switching: is there any biomechanical rationale?" Clinical Oral Implants Research, vol. 18, no. 5, pp. 581-584, 2007.

[15] R. J. Lazzara and S. S. Porter, "Platform switching: a new concept in implant dentistry for controlling postrestorative crestal bone levels," International Journal of Periodontics and Restorative Dentistry, vol. 26, no. 1, pp. 9-17, 2006.

[16] E. Nkenke, M. Hahn, K. Weinzierl, M. Radespiel-Tröger, F. W. Neukam, and K. Engelke, "Implant stability and histomorphometry: a correlation study in human cadavers using stepped cylinder implants," Clinical Oral Implants Research, vol. 14, no. 5, pp. 601-609, 2003.

[17] H.-S. Park, Y.-J. Lee, S.-H. Jeong, and T.-G. Kwon, "Density of the alveolar and basal bones of the maxilla and the mandible," American Journal of Orthodontics and Dentofacial Orthopedics, vol. 133, no. 1, pp. 30-37, 2008.

[18] M. P. Quesada-García, E. Prados-Sánchez, M. V. Olmedo-Gaya, E. Muñoz-Soto, M. P. González-Rodríguez, and M. VallecilloCapilla, "Measurement of dental implant stability by resonance frequency analysis: a review of the literature," Medicina Oral, Patologia Oral y Cirugia Bucal, vol. 14, no. 10, pp. e538-e546, 2009.

[19] O. K. Ahmad and J. R. Kelly, "Assessment of the primary stability of dental implants in artificial bone using resonance frequency and percussion analyses," The International Journal of Orale Maxillofacial Implants, vol. 28, no. 1, pp. 89-95, 2013.

[20] J.-T. Hsu, H.-L. Huang, C.-H. Chang, M.-T. Tsai, W.-C. Hung, and L.-J. Fuh, "Relationship of three-dimensional bone-toimplant contact to primary implant stability and peri-implant bone strain in immediate loading: microcomputed tomographic and in vitro analyses," The International Journal of Oral \& Maxillofacial Implants, vol. 28, no. 2, pp. 367-374, 2013.

[21] J.-T. Hsu, H.-L. Huang, M.-T. Tsai, A. Y.-J. Wu, M.-G. Tu, and L.-J. Fuh, "Effects of the 3D bone-to-implant contact and bone stiffness on the initial stability of a dental implant: micro-CT and resonance frequency analyses," International Journal of Oral and Maxillofacial Surgery, vol. 42, no. 2, pp. 276-280, 2013.

[22] T. Ohashi, S. Matsunaga, K. Nakahara et al., "Biomechanical role of peri-implant trabecular structures during vertical loading," Clinical Oral Investigations, vol. 14, no. 5, pp. 507-513, 2010.

[23] S. Matsunaga, Y. Shirakura, T. Ohashi et al., "Biomechanical role of peri-implant cancellous bone architecture," The International Journal of Prosthodontics, vol. 23, no. 4, pp. 333-338, 2010.

[24] R. Bernhardt, E. Kuhlisch, M. C. Schulz, U. Eckelt, and B. Stadlinger, "Comparison of bone-implant contact and boneimplant volume between 2D-histological sections and 3DSR $\mu$ CT slices," European Cells \& Materials, vol. 23, pp. 237-248, 2012.

[25] C. Makary, A. Rebaudi, G. Sammartino, and N. Naaman, "Implant primary stability determined by resonance frequency analysis: correlation with insertion torque, histologic bone volume, and torsional stability at 6 weeks," Implant Dentistry, vol. 21, no. 6, pp. 474-480, 2012.
[26] A. J. Wirth, J. Goldhahn, C. Flaig, P. Arbenz, R. Müller, and G. H. van Lenthe, "Implant stability is affected by local bone microstructural quality," Bone, vol. 49, no. 3, pp. 473-478, 2011.

[27] W.-J. Seong, U.-K. Kim, J. Q. Swift, J. S. Hodges, and C.-C. Ko, "Correlations between physical properties of jawbone and dental implant initial stability," Journal of Prosthetic Dentistry, vol. 101, no. 5, pp. 306-318, 2009.

[28] J.-T. Hsu, L.-J. Fuh, M.-G. Tu, Y.-F. Li, K.-T. Chen, and H.-L. Huang, "The effects of cortical bone thickness and trabecular bone strength on noninvasive measures of the implant primary stability using synthetic bone models," Clinical Implant Dentistry and Related Research, vol. 15, no. 2, pp. 251-261, 2013.

[29] T. M. G. J. van Eijden, "Biomechanics of the mandible," Critical Reviews in Oral Biology and Medicine, vol. 11, no. 1, pp. 123-136, 2000.

[30] F. Linde and H. C. F. Sorensen, "The effect of different storage methods on the mechanical properties of trabecular bone," Journal of Biomechanics, vol. 26, no. 10, pp. 1249-1252, 1993.

[31] C. N. Eliasa, F. A. Rocha, A. L. Nascimento, and P. G. Coelho, "Influence of implant shape, surface morphology, surgical technique and bone quality on the primary stability of dental implants," Journal of the Mechanical Behavior of Biomedical Materials, vol. 16, no. 1, pp. 169-180, 2012.

[32] D. O'Sullivan, L. Sennerby, and N. Meredith, "Measurements comparing the initial stability of five designs of dental implants: a human cadaver study," Clinical Implant Dentistry and Related Research, vol. 2, no. 2, pp. 85-92, 2000.

[33] A. M. O'Mahony, J. L. Williams, J. O. Katz, and P. Spencer, "Anisotropic elastic properties of cancellous bone from a human edentulous mandible," Clinical Oral Implants Research, vol. 11, no. 5, pp. 415-421, 2000. 


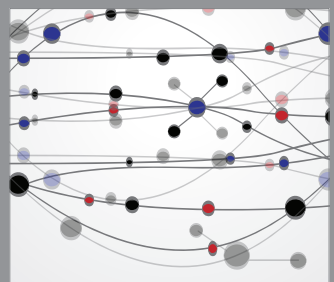

The Scientific World Journal
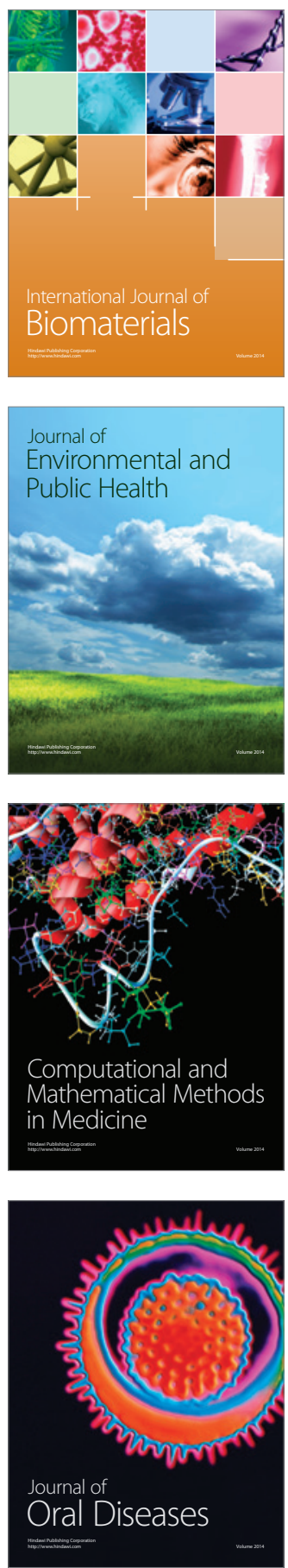
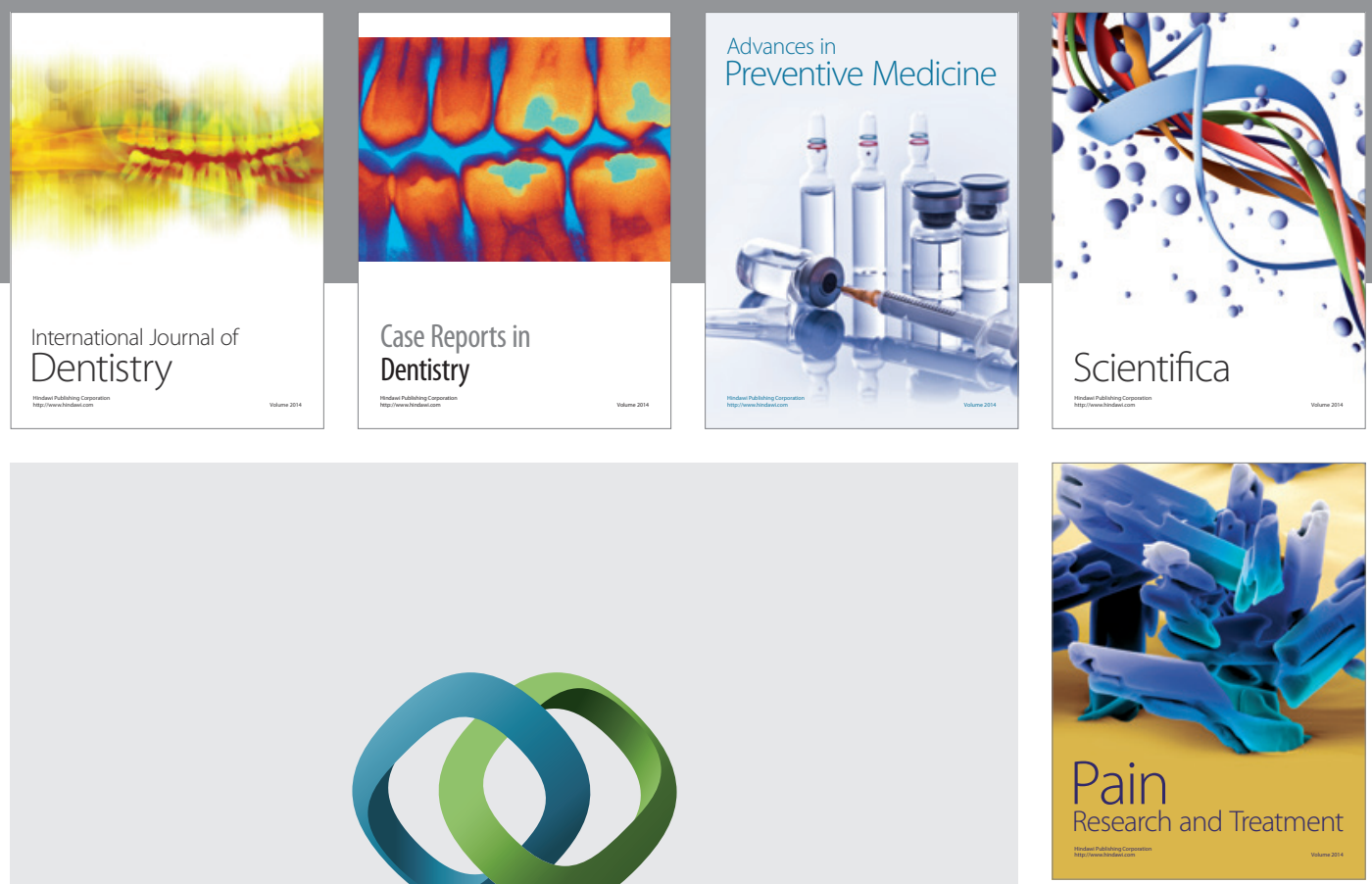

\section{Hindawi}

Submit your manuscripts at

http://www.hindawi.com
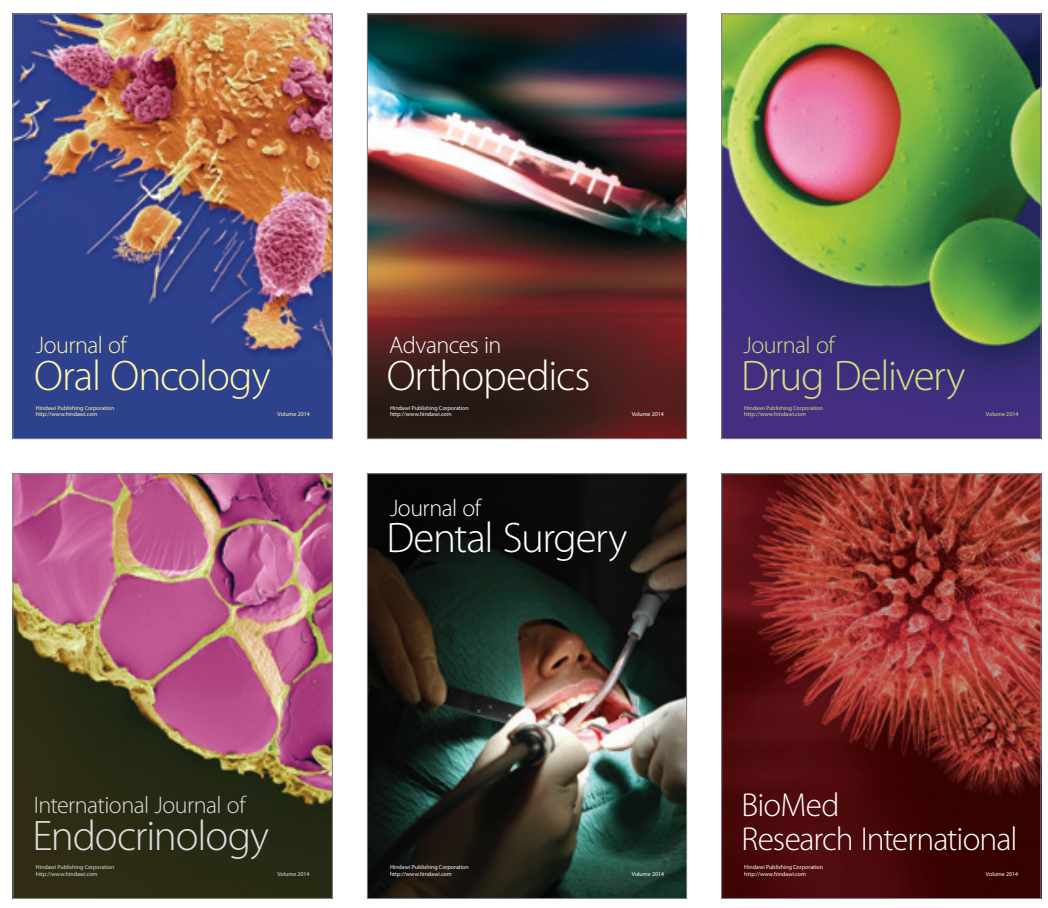

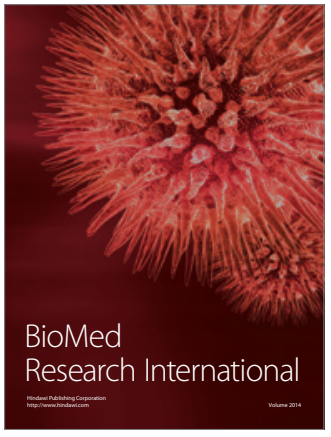

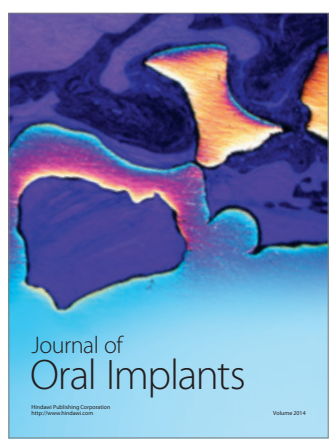
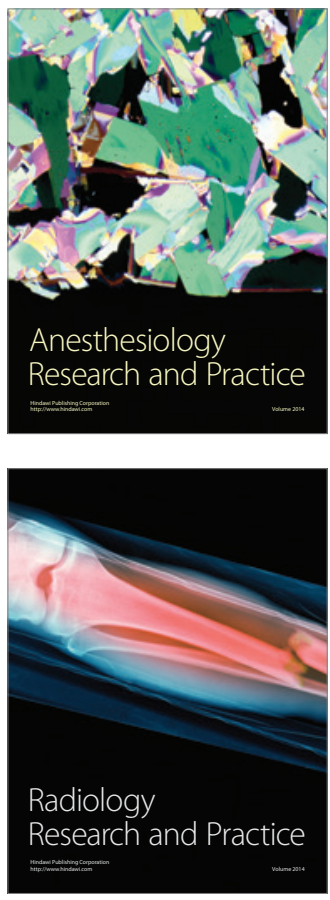